\section{A DICOTOMIA ENTRE TEORIA E PRÁTICA DO BRAINSTORMING}

\section{THE BRAINSTORMING DICHOTOMY BETWEEN THEORY AND PRACTICE}

\author{
Adriana Chammas ${ }^{1}$, M.Sc. \\ Manuela Quaresmaㄹ, D.Sc. \\ Cláudia Mont'Alvão ${ }^{3}$, D.Sc. \\ (1) Pontifícia Universidade Católica (PUC-Rio) \\ e-mail: ttdri@yahoo.com.br \\ (2) Pontifícia Universidade Católica (PUC-Rio) \\ e-mail: mquaresma@puc-rio.br \\ (3) Pontifícia Universidade Católica (PUC-Rio) \\ e-mail: cmontalvao@puc-rio.br
}

Criatividade, Ideação, Brainstorming.

Esse artigo contrapõe a prática atual do mercado tecnológico de desenvolvimento de produtos digitais com a fundamentação teórica que baliza a essência do Brainstroming. São apresentadas as adaptações mais relevantes através dos tempos e apresentados os resultados de 06 entrevistas com CEOs de grandes empresas desenvolvedoras e 66 respostas de um questionário específico que apontaram para a importância atemporal desta técnica.

\section{Creativity, Ideation, Brainstorming.}

This paper contrasts the current practice of the technological market for the development of digital products with the theoretical foundation that aims at the essence of Brainstorming. In addition, the most relevant adaptations through time are presented and the results of 06 interviews with CEOs of large companies and 66 responses of a specific questionnaire were presented, which pointed to the timeless importance of this technique.

\section{Introdução}

Este artigo ilustra a importância do Brainstorming através dos tempos ao destacar as alterações mais relevantes da técnica desde sua criação. A revisão teórica sobre a técnica foi contraposta com o discurso corrente dos profissionais do mercado de desenvolvimento tecnológico. Foram feitas 6 (seis) entrevistas com CEOs (Chief Executive Officers) de grandes empresas desenvolvedoras de produtos digitais e distribuídos 66 questionários online para desenvolvedores de produtos desse tipo. A pesquisa de campo deixou claro que adaptações são feitas de acordo com a realidade do projeto e/ou da empresa, mas que ainda assim o
Realização:

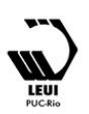




\section{$16^{\circ}$ \\ ERGODESIGN USIHC CINAHPA}

Brainstorming é, até hoje, uma das mais recorrentes e soberanas técnicas de ideação quando o intuito for inovar.

Diante da importância da tecnologia na rotina dos usuários e de toda praticidade advinda dela, o mercado tecnológico investe em produtos que incrementem a produtividade e pluralizem as possibilidades de seus usuários. Gui Bonsiepe (1997) há muito observa que o design está relacionado à inovação. Para o autor, design é a criatividade em forma de produção de novas ideias: "o design não tem sua origem em uma única pessoa, mas baseia-se num trabalho de equipe" [BONSIEPE, 1997].

Mas afinal... O que é inovação? Shavinina (2003) responde: "inovação é a geração, aceitação e implementação de novas ideias, produtos ou serviços".

Para Horn et al. (2014) inovação é a quebra de paradigmas para melhor. Kirton (2003) fez uma extensa revisão da literatura que aponta a inovação como o estágio final de implementação da criatividade. $\mathrm{O}$ autor discute que inovação e criatividade não são sinônimos e que se a inovação sempre estiver associada à implementação, a criatividade será fadada à geração de ideias: "criatividade é subconjunto da solução do problema" [KIRTON, 2003]. Inovação, para o autor, é a solução de um problema com os recursos que permitem (e promovem) futuras soluções de novos problemas. Inovação nada mais é do que o produto dos inovadores. Em contraste com Bonsiepe (1997), Amabile (1988, 1996) acredita que a inovação se origina individualmente. De acordo com Amabile, Shavinina (2003) entende que o inovador é um indivíduo com experiência cognitiva diferenciada e integrada, que aproveitou os períodos sensitivos de sua infância para otimizar sua experiência cognitiva e transformá-la em uma forma única de ler, interpretar e formar uma representação intelectual única do mundo - que se expressa na habilidade de gerar ideias. Já Bonsiepe (1997) e Dyer et al. (2011) afirmam que a inovação industrial - e, por analogia a tecnológica, devem ocorrer em equipes. Entende-se, a partir do exposto, que a inovação na indústria é resultante da reunião de vários criativos individuais em busca de uma solução comum. $16^{\circ}$ Ergodesign - Congresso Internacional de Ergonomia e Usabilidade de Interfaces Humano Tecnológica: Produto, Informações Ambientes Construídos e Transporte

$16^{\circ}$ USIHC - Congresso Internacional de Ergonomia e Usabilidade de Interfaces Humano Computador

CINAHPA | 2017 - Congresso Internacional de Ambientes Hipermídia para Aprendizagem.

Dyer et al. (2011) apontam que equipes inovadoras podem até apresentar algumas diferenças entre os talentos individuais, mas que as diferenças são diluídas nos resultados do grupo. Não por acaso Kelley \& Littman (2005) categorizam o Brainstorming como ponto de partida para a inovação, pois "é divertido, liberta altas doses de energia e é capaz de gerar resultados com mais rapidez do que qualquer outra técnica". Para eles, uma cultura de inovação deve partir de uma cultura de Branstormings recorrentes, assim talentos individuais potencializarão a criatividade de todos. Publicitário e pesquisador interessado no poder criador da mente, Alex Osborn (1942, 1953, 1957, 1963, 1975) criou e desenvolveu o Brainstorming nos anos 40s, uma técnica exploratória que incentiva novas propostas, possibilidades e significados. As ideias surgem a partir da exploração de determinado tema por um grupo específico, reunido sob condições específicas. A literatura sobre o Brainstorming investiga principalmente formas de incrementar a produtividade - e em última análise, a inovação.

\section{Origens e Descrição da Técnica}

Referência no tocante à inovação, o Brainstorming foi ponto de partida para o desenvolvimento de muitas outras técnicas de geração de ideias, a exemplo do Brainwriting, do Braindrawing ${ }^{l}$ e do Grupo de Foco, criado na mesma época. Como pontos positivos do Brainstorming elencam-se a simplicidade, o baixo custo, os resultados rápidos, a possibilidade de fazer combinações e estender ideias, a fomentação da criatividade e da inovação.

O trabalho de Alex Osborn (1942, 1953, 1957, 1963, 1975) deixa claro que, à excessão dos princípios fundamentais, o Brainstorming não é uma técnica inflexível e que deve se adaptar às circunstâncias, mas afirma que o Brainstorming necessariamente deve seguir os seguintes

\footnotetext{
${ }^{1}$ Ambas técnicas de ideação rápida similares ao Brainstorming. As técnicas se utilizam de ideias escritas e de desenhos, respectivamente, para que os demais integrantes tenham mais ideias.
}

Realização:

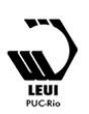




\section{$16^{\circ}$ \\ ERGODESIGN USIHC CINAHPA}

princípios:

1. A crítica deve ser proibida: "a ideação torna-se mais produtiva quando se exclui a crítica exercida simultaneamente, já que devido à educação e à experiência, muitos adultos foram treinados a pensar mais judiciosamente do que 'criadoramente"' (SIC); Julgamentos adversos às ideias devem ser colocados mais tarde;

2. Encorajar o "Freewheeling 2": o participante deve falar toda ideia que lhe vier à mente, quanto mais "descabida" a ideia, melhor;

3. A quantidade de ideias é preferível à qualidade delas: "na ideação, a quantidade contribui para melhorar a qualidade"; Quanto maior a quantidade de ideias, maior a proporção de ideias úteis.

4. Em determinado momento da sessão deve-se buscar a combinação e refino, ou seja, participantes devem sugerir como a ideia dos demais membros do grupo podem se tornar melhores, ou como duas ou mais ideias podem ser somadas e formarem uma terceira.

Em nome da eficácia da técnica, Osborn (1953, 1957) recomenda que: intervalos aconteçam durante a sessão, objetivos específicos sejam apresentados ao grupo de participantes e que o moderador seja bem treinado. Ao publicar "O Poder Criador da Mente: Princípios e Processos do Pensamento Criador e do Brainstorming", Osborn (1975) confirmou a eficácia de sua técnica ao descrever vários estudos de caso sobre o Brainstorming. A partir deles, o autor elencou o procedimento ideal da técnica:

(a) a composição do grupo, que deve ser de um moderador, um ajudante, cinco participantes com familiaridade ao projeto e cinco outros, convidados. Deve ser convidado um grupo diferente para cada sessão. Deve-se atentar para o equilíbrio do grupo para que problemas

\footnotetext{
${ }^{2}$ A expressão "Freewheeling" pode ser traduzida do inglês como um ato praticado sem preocupação com regras, convenções ou consequências.
}

$16^{\circ}$ Ergodesign - Congresso Internacional de Ergonomia e Usabilidade de Interfaces Humano Tecnológica: Produto, Informações Ambientes Construídos e Transporte

$16^{\circ}$ USIHC - Congresso Internacional de Ergonomia e Usabilidade de Interfaces Humano Computador

CINAHPA | 2017 - Congresso Internacional de Ambientes Hipermídia para Aprendizagem.

relacionados à hierarquia, por exemplo, possam interferir no fluxo de ideias;

(b) o moderador deve ser treinado para exercer sua função. Uma vez entendidos os princípios do Brainstorming, o moderador deve ter em seu poder uma lista com sugestões de soluções para o problema que for apresentado na sessão, para que, caso o fluxo de ideias esmoreça, o moderador possa ativá-lo novamente com sugestões. Deve-se deixar claro que o moderador deve se conter ante à fala de qualquer outro participante. $\mathrm{O}$ ajudante não interfere na sessão, somente apoia o moderador e se encarrega dos registros. As ideias devem ser anônimas;

(c) dois dias antes da sessão os participantes devem receber um esquema, um esboço, algum material sobre o assunto a ser colocado na sessão para que possam refletir sobre o tema, mas que não revele o problema em si;

(d) o autor sugere que seja servido um lanche antes da sessão, para a sociabilização dos participantes do grupo.

(e) dá-se início à sessão e, ainda na apresentação, o moderador deve fazer um aquecimento, com exercícios fáceis e simples de ideação;

(f) o moderador deve fazer então uma classificação (ou categorização), para dar rumo à sessão: "a orientação do alvo é muitas vezes meia batalha ganha" [OSBORN, 1975];

(g) ao apresentar o problema real, o moderador deve partir da premissa que o problema (ou desafio) a ser solucionado deve primar pelo particular (e não pelo geral) e que deve ser simples. Em caso de problemas complexos, dividi-los em subproblemas;

(h) o moderador deve apresentar os quatro princípios fundamentais do Brainstorming, que deve estar afixados e à vista do grupo durante toda a sessão;

(i) o moderador deve pedir sugestões para os participantes de forma que cada membro apresente uma única ideia de cada vez - o autor 


\section{$16^{\circ}$ \\ ERGODESIGN USIHC CINAHPA}

$16^{\circ}$ Ergodesign - Congresso Internacional de Ergonomia e Usabilidade de Interfaces Humano Tecnológica: Produto, Informações Ambientes Construídos e Transporte

$16^{\circ}$ USIHC - Congresso Internacional de Ergonomia e Usabilidade de Interfaces Humano Computador

CINAHPA | 2017 - Congresso Internacional de Ambientes Hipermídia para Aprendizagem. recomenda que os participantes tomem nota das ideias que possam surgir para evitar que a esqueçam enquanto não lhes chega a vez;

(j) ao se ter por produto cerca de uma centena de ideias, o moderador deve agradecer a participação do grupo e pedir-lhes que conservem a sessão em mente para que ainda possam contribuir, se contatados a posteriori.

Osborn (1975) esclarece que a qualidade das ideias está diretamente relacionada aos processos subsequentes, tais como a discussão, seleção, combinação etc. Não raro o Brainstorming é confundido com um processo completo de solução de problemas / desafios de forma criativa, quando na verdade a técnica é apenas parte do processo cunhado por Osborn e revisto por ele e Sid Parnes - o CPS (Creative Problem Solving). O

Brainstorming, conforme classificação de ambos os autores citados, é a fase divergente do processo criativo - e pode ser feito de forma individual, colaborativa ou em grupo.

\section{Pontos Fomentadores de Pesquisas Sobre o Brainstorming}

Das questões que mais suscitaram pesquisas, a mais popular dentre os registros estudados foi a questão de o Brainstorming ser em grupo ou individual [DIEHL \& STROEBE, 1987, 1991; MULLEN et al, 1991, CAMACHO \& PAULUS, 1995]. Esse e os demais pontos elencados aqui serão especificados na seção seguinte, onde serão apresentadas as adaptações feitas.

De acordo com Dugosh \& Paulus (2004), Osborn entende que a efetividade do Brainstorming está relacionada tanto aos processos cognitivos (associação, por exemplo) quanto aos sociais (sociabilização, competição etc.). De acordo, pesquisas psicológicas recentes [GOLDENBERG \& WILEY, 2011] têm concluído que, no caso do Brainstorming em grupo, a criatividade tende a ser tolhida, já que as conexões necessárias para criar podem ser inibidas pelas reticências em expressar um pensamento divergente frente ao grupo. Outros aspectos que devem ser levados em conta são o comportamento de determinados membros do grupo, a hierarquia entre os participantes, as divergências sociais etc. Dugosh et al. (2000, 2004) e Goldenberg \& Wiley (2011) discutem os efeitos da estimulação cognitiva do Brainstorming, bem como a comparação social entre os participantes de um mesmo grupo. Os autores discutem as vantagens e atentam para desvantagens potenciais, como a conformidade ou a fixação perante os demais participantes especialmente os de diferentes classes sociais. Quanto ao procedimento ideal, foram levantadas dúvidas pelos pesquisadores da época, William J J Gordon e Artur D. Little apud Gobble (2014), sobre a ausência de criticismo em todo o Brainstorming. Outro ponto relevante comentado por J.P. Guilford, ainda nos anos 1950s, diz respeito à seguinte afirmação de Osborn (1975): o problema a ser solucionado deve primar pelo particular e não pelo geral.

Bezzi (2011) propôs uma variação do Brainstorming ao somar a análise e exploração do espaço semântico das ideias pelo grupo. Gobble (2014) aponta que existem abordagens alternativas que preterem o "Freewheeling" incentivado pelo Brainstorming clássico em nome de maior estruturação no processo de resolução de problemas. A autora reconhece a importância do Brainstorming, mas coloca que existem limitações na técnica. Rossiter e Lilien (1994) e Paulus et al. (2006, 2011) exploraram o efeito das instruções e a importância do moderador nos resultados da sessão.

\section{Adaptações Relevantes}

Uma vez elencados os pontos acima, seguem as adaptações de maior relevância feitas através dos tempos, para adequar a técnica de acordo com as limitações encontradas pelos autores. William J J Gordon e Artur D. Little criaram o Sinectics [GOBBLE, 2014], que expande um dos princípios do Brainstorming original, o de suspender julgamentos, por um processo mais elaborado. $\mathrm{O}$ Sinectics inicia com a apresentação (sem críticas) do problema definido pelos responsáveis pelo projeto (owners) e faz com que os participantes o recoloquem de acordo com seu entendimento. A partir de então, os participantes listam todas as soluções encontradas para o problema. $\mathrm{O}$ moderador então redireciona o grupo a uma sessão de Brainstorm na qual, diferentemente do
Realização:

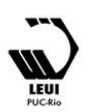




\section{$16^{\circ}$ \\ ERGODESIGN USIHC CINAHPA}

Brainstorming original, o moderador introduz metáforas e o imaginário na discussão de forma estruturada, para encorajar novas conexões intuitivas. A técnica adiciona um passo ao brainstorming tradicional ao incluir o desenvolvimento da ideia e somar ao grupo dos participantes, os responsáveis pelo projeto (owners). Ainda em meados dos anos 1950s, J.P. Guilford (1950) incorporou aos princípios de Osborn a orientação de "divergir antes de convergir". A lógica do pensamento divergente é a geração de ideias, enquanto a do pensamento convergente é a avaliação e consequente decisão a respeito das opções, em busca das ideias ideais. Para o autor, quando ideias suficientes tiverem sido geradas deve-se promover o debate e a crítica, a comparação e o descarte de ideias irrelevantes, impraticáveis etc.

Mais tarde Osborn introduziu o CPS (Creative Problem-Solving) em conjunto com Sidney J. Parnes (1959), cuja primeira versão consiste de encontrar três pontos básicos: fato, ideia e solução. Encontrar o fato diz respeito à definição do problema e analisar os dados pertinentes a ele. Encontrar a ideia relaciona-se com a produção de ideias em si, tanto o pensamento que o autor chama de "sublime" quanto o desenvolvimento da ideia (seleção, processamento, modificações e combinações); Encontrar a solução abarca a avaliação (verificação das tentativas de solução) e da adoção (decisão e implementação da solução final). Cada um desses estágios envolve um Brainstorming independente, ou seja, o Brainstorming é sempre o pensamento divergente no processo criativo. O CPS abarca três estágios conceituais, onde a abordagem é feita de fora para dentro. São propostas seis repetições de divergência / convergência e um executivo (responsável pelo projeto) modera a atividade. $\mathrm{O}$ CPS é composto de sete passos [OSBORN, 1953]: (a) orientação, quando aponta-se para o problema; (b) preparação, quando adquirem-se dos dados disponíveis e pertinentes; (c) análise: quando disseca-se o material relevante; (d) hipótese: quando viabilizam-se alternativas dentro das ideias propostas; (e) incubação: quando incentiva-se o que ideias "iluminadas" ocorram; (f) síntese: quando as melhores ideias são somadas, associadas e combinadas; (g) verificação: julgamento das ideias resultantes. Passados mais de 40 anos de $16^{\circ}$ Ergodesign - Congresso Internacional de Ergonomia e Usabilidade de Interfaces Humano Tecnológica: Produto, Informações Ambientes Construídos e Transporte

$16^{\circ}$ USIHC - Congresso Internacional de Ergonomia e Usabilidade de Interfaces Humano Computador

CINAHPA | 2017 - Congresso Internacional de Ambientes Hipermídia para Aprendizagem.

pesquisa, a evolução do CPS continua ativa. Junto com outros autores, Sidney J. Parnes, sócio de Osborn e co-fundador do Creative Education Foundation, propôs releituras do processo do CPS.

Debruçados em mais de quarenta anos de pesquisa sobre o Brainstorming, Rossiter \& Lilien (1994) geraram seis novos princípios para o Brainstorming que consideram essenciais quando o intuito for a geração de ideias:

1. As instruções do Brainstorming clássico de Osborn (1953) são essenciais para o sucesso da técnica, em especial a que pretere a qualidade em nome da quantidade de ideias;

2. Baseados nos experimentos de Wood et al. (1987), Earley \& Lituchy (1991) e Wood et al. (1992), Rossiter \& Lilien (1994) estatizam que deve ser proposto um número de ideias a serem produzidas na sessão. O número deve ser específico e difícil de ser atingido;

3. As ideias iniciais devem ser propostas por indivíduos, não grupos. Os indivíduos devem trabalhar sozinhos pelo menos no estágio de ideação inicial. De acordo com Rossiter \& Lilien (1994), quanto maior o grupo, menor o número de ideias, se comparadas ao número de ideias produzidas individualmente;

4. A interação entre o grupo deve ser usada para somar e refinar as ideias geradas individualmente. De acordo com Delbecq et al. (1986), Rossiter \& Lilien (1994) orientam que as ideias devem ser expostas numa lista anônima, com igual tempo de discussão para cada uma. Os participantes devem expor as razões que os levaram a concordar ou não com cada ideia.

5. As ideias devem ser selecionadas por votação individual, onde os votos dos participantes têm igual peso.

6. O tempo dado para a geração de ideias iniciais deve ser curto (15 minutos).
Realização:

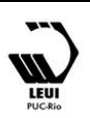




\section{$16^{\circ}$ \\ ERGODESIGN USIHC CINAHPA}

$16^{\circ}$ Ergodesign - Congresso Internacional de Ergonomia e Usabilidade de Interfaces Humano Tecnológica: Produto, Informações Ambientes Construídos e Transporte

$16^{\circ}$ USIHC - Congresso Internacional de Ergonomia e Usabilidade de Interfaces Humano Computador

CINAHPA | 2017 - Congresso Internacional de Ambientes Hipermídia para Aprendizagem.
Rossiter \& Lilien (1994) enfatizam que o problema deve estar claramente definido e devidamente apresentado ao moderador da sessão. Os autores lembram que uma definição imprecisa pode direcionar as ideias do grupo à improdutividade.

Quanto ao processo de incubação, Olton (1979) e Read et al.(1982) apud Rossiter \& Lilien (1994) discordam de achados anteriores ao colocarem que não foram encontradas evidências convincentes de que a incubação é um processo autônomo inconsciente. A neurociência recente aponta que a criatividade ocorre em todo o cérebro, num complexo processo de ligações que carece de tempo e espaço para despontar. De acordo, Wallas (1926) apud Rossiter \& Lilien (1994) em seu modelo de pensamento criativo composto de quatro fases (preparação, incubação, iluminação e verificação) defende que o período de "incubação" é absolutamente necessário. Nessa lógica a criatividade pode não ocorrer somente no curto espaço de tempo de um Brainstorming, mas ao solicitar que os participantes chegassem às sessões com o tema do Brainstorming em mente e preparálos para um possível contato a posteriori, Osborn (1953) demonstrou ser visionário ao entender da mesma forma. De acordo com o exposto, acreditase que a incubação assume papel crucial nessa técnica de ideação.

Em 2006, Cláudio Bezzi (2006) propôs o Evaluational Brainstorming, uma adaptação do Brainstorming clássico que soma ao compasso tradicional da aplicação da técnica, a análise e exploração do espaço semântico das ideias pelo grupo. Através da categorização e construção de mapas semânticos das ideias condutoras, $\mathrm{o}$ entendimento compartilhado possibilita averiguar se os objetivos da aplicação da técnica serão alcançados. As etapas do Evaluational Brainstorming são as seguintes: A fase criativa, conforme o Brainstorming clássico; a fase de classificação, um processo indutivo; a fase de sintetização, um processo dedutivo. A abordagem gera o pensamento indutivo do grupo e o conecta a um processo de comunicação pragmático. Isso gera uma significação particular dentro do contexto do conceito explorado.

A mais conhecida das abordagens alternativas que preterem o "Freewheeling" é o Triz Method, ou método $\mathrm{TRIZ}^{3}$, adotado por empresas como Google, Apple, General Motors, Johnson \& Johnson, Ford, Motorola, Procter \& Gamble, Xerox entre outras. O TRIZ é um método de resolução de problemas e desenvolvimento de produtos inovadores criado pelo cientista Russo Genrich Altshuller (1926-1998) apud Livotov (2008) que, autor de mais de 70 invenções patenteadas, adaptou o método TRIZ à prática gerencial. O método contrasta com tantos outros, calcados na tentativa-erro para resolver problemas e criar produtos inovadores. Baseado em leis imparciais sobre a evolução dos sistemas, o TRIZ faz uma busca sistêmica por soluções. O TRIZ identifica, exacerba e elimina as contradições técnicas (também chamadas propriedades contraditórias técnicas) de processos ou sistemas completamente baseado na lógica. $\mathrm{O}$ método sistemático de inovação apresenta uma sorte de variações da ferramenta, sendo a mais comum para identificar e resolver contradições nos sistemas ou processos, os 40 princípios inventivos (IPs)

[GAZEN \& RAHMAN, 2014]. Cada IP consiste em sub-princípios que se expandem em outros princípios e, de fato, produzem soluções inovadoras ao resolver problemas de contradição do sistema ou do processo.

Gobble (2014) lembra que o fio condutor do TRIZ é a argumentação e a recombinação. Alguém, em algum lugar, já se deparou com problema semelhante, o que significa que encontrar a solução correta para determinado problema significa encontrar outra solução previamente desenvolvida que se adeque à solução do problema em questão. Os participantes saem da especificidade do problema particular para a generalização, então voltam para a especificidade da solução do problema particular. O método, não poderia deixar de ser mencionado, já que é utilizado em empresas de tecnologia de ponta em

\footnotetext{
${ }^{3} \mathrm{O}$ nome do método, TRIZ, é um acrônimo em russo, que significa "Theoria Resheneyva Isobretatelskehuh Zadach". Sua tradução é "teoria inventiva de resolução de problemas".
}

Realização:
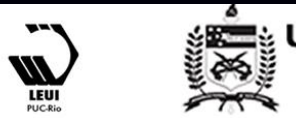


\section{$16^{\circ}$ \\ ERGODESIGN USIHC CINAHPA}

busca de inovação. O método TRIZ baseia-se em operações lógicas sem ponderar a subjetividade envolvida no Design do desenvolvimento de produtos digitais. Dessa forma, entende-se que, apesar de importante modelo do tópico inovação, o método TRIZ é mais pertinente à inovação tecnológica do que à inovação em Design. Além do TRIZ, Gobble (2014) considera a quantidade de métodos para estimular a criatividade e provocar a inovação. Muitos deles combinam elementos de teorias sobre o funcionamento da criatividade humana. Ocorre que, para a autora, não existe uma única técnica de ideação ideal, já que devem ser ponderados o tipo de problema, de pessoas e o contexto no qual ele estiver inserido. A autora ressalta a importância do Brainstorming, mas aconselha que adaptações sejam feitas, de acordo com a demanda específica do projeto.

As sugestões de Gobble (2014) são as que seguem: (a) os participantes devem fazer seu Brainstorming individual, para só então trazer as ideias para o grupo. Dessa forma os participantes podem exercer o poder da criatividade individual e potencializá-lo com a colaboração e combinação com as ideias dos demais integrantes do grupo;

(b) as sessões de Brainstorming devem ser mais estruturadas, de forma a incrementar a produtividade. Devem ser apresentadas questões em ordem estruturada, bem como propostas atividades específicas;

(c) as contribuições individuais devem então ser registradas de forma visível para possibilitar uma nova cascata de associações;

(d) a prática do moderador e a qualidade da condução do Brainstorming são diretamente relacionadas ao sucesso: o moderador deve criar a mágica entre os participantes, facilitando o surgimento das ideias no grupo;

(e) os participantes já devem ter feito o Brainstorming individual antes da sessão, para minimizar a pressão do grupo ou a fixação cognitiva, por consequência, aprimorar o processo colaborativo. $16^{\circ}$ Ergodesign - Congresso Internacional de Ergonomia e Usabilidade de Interfaces Humano Tecnológica: Produto, Informações Ambientes Construídos e Transporte

$16^{\circ}$ USIHC - Congresso Internacional de Ergonomia e Usabilidade de Interfaces Humano Computador

CINAHPA | 2017 - Congresso Internacional de Ambientes Hipermídia para Aprendizagem.

\section{O Brainstorming na Prática}

Para entender a prática do Brainstorming pelo mercado, foram feitas 6 (seis) entrevistas presenciais com os CEOs de grandes empresas desenvolvedoras de produtos digitais entre os meses de abril a novembro de 2015. A intenção da escolha de CEOs foi trazer diferentes enfoques sobre a ideação no processo de desenvolvimento, entender se e como o Brainstorming é utilizado e qual sua importância no processo, enfim, entender o ponto de vista gerencial sobre a técnica. As entrevistas semi-estruturadas focalizadas foram o tipo de entrevista que melhor se adequou ao perfil. As entrevistas partiram de um roteiro de ordem e redação flexível, embora todos os tópicos tenham sido abordados. A formulação das perguntas teve critérios simples e diretos para facilitar a comunicação entre entrevistado e entrevistador e provocaram respostas abertas e reflexivas. Foi aplicado um teste piloto para minimizar os possíveis percalços durante a aplicação da técnica com um dos sócios de uma empresa de Design focada em produtos digitais. Foram apresentados e assinados pelos participantes termos de consentimento antes desta pesquisa de campo. $\mathrm{O}$ teste piloto apontou para a reordenação das perguntas apenas e a seguir, foi desconsiderado.

Para permitir entender a importância e a prática do Brainstorming pelo mercado sob a ótica operacional $l^{4}$, tanto a praticada, quanto a idealizada pelos desenvolvedores, foi aplicado um questionário composto de 12 questões abertas e de 14 questões fechadas, orientadas às práticas e opiniões sobre o processo de desenvolvimento dos produtos, em especial sobre a ideação. $\mathrm{O}$ questionário foi validado a partir do teste piloto com 5 (cinco) usuários. Os testes piloto foram analisados e descartados a seguir. Foi necessária a reformulação de apenas duas das questões e comprovada a eficácia da aplicação da técnica para esse propósito. O questionário ficou disponível online, de 27 de abril a 27 de junho de 2015 . foi acessado 278 vezes e angariou 66 respostas válidas

4 Entende-se por ótica operacional a visão dos desenvolvedores e demais envolvidos na equipe. 


\section{$16^{\circ}$ \\ ERGODESIGN USIHC CINAHPA}

até a data limite. A construção do questionário foi inspirada nos mesmos parâmetros que balizaram o roteiro das entrevistas com os CEOs.

As seis entrevistas nomearam o Brainstorming como ferramenta indispensável quando o intuito for a inovação em produtos digitais. A totalidade dos entrevistados confirmou o uso da técnica e reafirmou que adaptações são feitas em virtude dos projetos, mas nenhum deles verbalizou algum modelo que fosse replicável a todos (ou a grande parte) os projetos. Consenso geral, a prática do Brainstorming não é necessariamente formalizada, tão pouco nomeada. O Brainstorming é uma técnica natural, quase orgânica dentro do processo e as adaptações não são idênticas em todos os casos.

Nos resultados da aplicação do questionário, apenas 3 (três) dos respondentes disseram utilizar o Brainstorming como uma das técnicas de ideação no processo de desenvolvimento, embora 30 dos 66 respondentes tenha ressaltado a etapa de criação no processo. Embora a etapa de criação não tenha sido especificamente esmiuçada, entende-se, em meio às demais respostas do questionário e os resultados das entrevistas, que o Brainstorming não só faça parte da etapa de criação, mas que tenha expressiva importância dentro do processo. Fato que corrobora esse entendimento, 32 respondentes do questionário descreveram parcialmente o Brainstorming sem nomeá-lo, ao responderem sobre como estimular a criatividade.

\section{Conclusão}

Entende-se que a produção de ideias criativas é imperativa para a geração de novos conceitos, de novos produtos, da inovação e consequentemente vital para o mercado. Ainda que o Brainstorming tenha fomentado pesquisas que desencadearam adaptações à técnica, é utilizado de forma recorrente até hoje e já deu origem a diversas outras técnicas de inovação. A pluralidade dos desdobramentos da técnica, a exemplo do Sinectics, que propõe repensar um dos princípios básicos do Brainstorming (o de não julgar) ou a franca exploração do espaço semântico das ideias (Evaluational Brainstorming), indica que a maleabilidade da técnica é ponto positivo - e previsto pelo seu criador. Reconhece-se que o $16^{\circ}$ Ergodesign - Congresso Internacional de Ergonomia e Usabilidade de Interfaces Humano Tecnológica: Produto, Informações Ambientes Construídos e Transporte

$16^{\circ}$ USIHC - Congresso Internacional de Ergonomia e Usabilidade de Interfaces Humano Computador

CINAHPA | 2017 - Congresso Internacional de Ambientes Hipermídia para Aprendizagem.

Brainstorming em grupo não é a melhor solução para a propositura de novas ideias - embora seja para refiná-las. Entende-se que o grupo deve trabalhar individualmente antes de se reunir para evitar a queda da produtividade com a pressão entre os pares - ou a fixação cognitiva, por exemplo, já que em atmosferas rígidas a criatividade pode ser inibida. Conclui-se que dessa forma a carga cognitiva dos participantes também será aliviada.

Conclui-se também, após a contraposição da literatura, que Brainstorming individual deva acontecer no início e no fim do processo, enquanto o Brainstorming em grupo deve acontecer no meio dos dois extremos. Outro ponto relevante e fácil de ser percebido na evolução do Brainstorming através dos tempos é a importância da experiência e da expertise do moderador ao aplicar a técnica.

Entende-se que o papel do moderador é imperativo para o sucesso do Brainstorming.

O Brainstorming mostrou-se mais flexível na prática do que na literatura examinada. Muitos desenvolvedores fazem uso da técnica (ainda que resumidamente) sem ao menos perceber, por já a terem incutida no processo - e também pela falta de formalidade na aplicação, já que cada projeto demanda seu próprio modelo de Brainstorming. Tanto os desenvolvedores quanto os CEOs entrevistados demonstraram entender 0 Brainstorming apenas como a reunião de algumas pessoas para discutir determinado assunto, sem compromisso com a viabilidade das ideias. Em nenhum dos casos ficou clara a sapiência dos preceitos clássicos da técnica de Osborn (1942, 1953, 1957, 1963, 1975) ou de qualquer das adaptações apresentadas neste artigo. Ocorre que os créditos do Brainstorming podem acabar por se perder e essa técnica simples, de baixo custo, que promove resultados rápidos e fomenta a criatividade e a inovação, pode ser subutilizada sem o embasamento teórico na prática.

A partir do exposto entende-se que o mercado poderia se valer da fundamentação teórica da técnica e das adaptações já consagradas pela literatura para obter um modelo maleável para o processo, mas que não se perdesse em meio aos fatores limitantes de cada realidade. A própria difusão dos preceitos do Brainstorming mostrou-se
Realização:

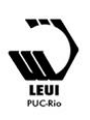




\section{$16^{\circ}$ \\ ERGODESIGN USIHC CINAHPA}

relevante na prática. Pontos como os discutidos neste artigo, a exemplo da etapa de criação individual, entre tantos outros, devem acontecer durante o Brainstorming para que os resultados esperados sejam alcançados, quiçá superados.

Ainda que o Brainstorming tenha tido que passar por um processo evolutivo através dos tempos, não há como negar sua importância. A quantidade e qualidade dos desdobramentos suscitados por ele, seja clássico ou adaptado, prático ou teórico, apontam que o Brainstorming é ferramenta vital para o processo de inovação. Entende-se que esse método, desde que corretamente aplicado, seja ideal para captar as melhores ideias e fazer delas ideais.

\section{BIBLIOGRAFIA}

AMABILE, T. M. A model of creativity and innovation in organizations. In B. M. Staw \& L. L. Cummings (Eds.), Research in organizational behavior, Vol. 10, 123-167. Greenwich, CT: JAI Press, 1988.

AMABILE, Teresa M.; Creativity in Context Update to the Social Psychology of Creativity. Boulder, CO: Westviewpress, 1996.

BYRON, Kevin. Creative Reflections on Brainstorming. London Review of Education, vol 10, No2, July 2012, 201-213.

BONSIEPE, Gui. Design: do material ao digital. Tradução de Cláudio Dutra. Florianópolis:

FIESC/IEL, 1997.

CAMACHO, L. M., \& Paulus, P. B. (1995). The role of social anxiousness in group brainstorming. Journal of Personality and Social Psychology, 68, 1071-1080.

DELBECQ AL, Van de Ven AH (1971) A group process model for problem identification and program planning. J. Appl. Behavioral Sci. 7(4):466-492.

DIEHL, M., \& STROEBE, W. (1987).

Productivity loss in brainstorming groups: Toward the solution of a riddle. Journal of $16^{\circ}$ Ergodesign - Congresso Internacional de Ergonomia e Usabilidade de Interfaces Humano Tecnológica: Produto, Informações Ambientes Construídos e Transporte

$16^{\circ}$ USIHC - Congresso Internacional de Ergonomia e Usabilidade de Interfaces Humano Computador

CINAHPA | 2017 - Congresso Internacional de Ambientes Hipermídia para Aprendizagem.

Personality and Social Psychology, 53, 497-509.

DIEHL, M., \& STROEBE, W. (1991).

Productivity loss in idea-generating groups:

Tracking down the blocking effect. Journal of

Personality and Social Psychology, 61, 392-403.

DYER, Jeff; GREGERSEN, Hal.;

CHRISTENSEN, Clayton M. The innovator's

DNA : mastering the five skills of disruptive innovators. HARVARD BUSINESS REVIEW PRESS: BOSTON, 2011.

DUGOSH, Karen L., PAULUS, Paul, B., ROLAND, Evelyn J., YANG, Huei-Chuang. Cognitive Stimulation in Brainstorming. Journal of Personality and Social Psychology, 2000. Vol. 79, No. 5, $722-735$ the American Psychological Association, Inc

DUGOSH, Karen L., PAULUS, Paul, B. Cognitive and Social Comparision Process in Brainstorming. Journal of Experimental Social Psychology 41 (2005) 313-320

GUILFORD, J. Creativity. American Psychologist 5: 444-54 1950

GOBBLE, MaryAnne M. The Persistence of Brainstorming. Research-Technology Management, January - February 2014.

GOBBLE, MaryAnne M. Beyond Brainstorming. Research-Technology Management, March- April 2014.

GOLDENBERG, O. \& WILEY, J. Quality, Conformity, and Conflict: Questioning the Assumptions of Osborn's Brainstorming Technique. The Journal of Problem Solving, volume $3, \mathrm{n}^{\circ} 2$ (winter 2011)

KELLEY, T.; LITTMAN, J.: The Ten Faces of Innovation. IDEO's Strategies for Defeating the Devil's Advocate and Driving Creativity Throughout Your Organization. Doubleday, 2005.

KIRTON, M. Adaption-innovation: In the context of diversity and change. New York, NY: Routledge., 2003.

MULLEN, B., Johnson, C, \& Salas, E. (1991). Productivity loss in brainstorming groups: A
Realização:

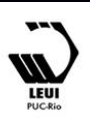




\section{$16^{\circ}$ \\ ERGODESIGN USIHC CINAHPA}

$16^{\circ}$ Ergodesign - Congresso Internacional de Ergonomia e Usabilidade de Interfaces Humano Tecnológica: Produto, Informações Ambientes Construídos e Transporte

$16^{\circ}$ USIHC - Congresso Internacional de Ergonomia e Usabilidade de Interfaces Humano Computador

CINAHPA | 2017 - Congresso Internacional de Ambientes Hipermídia para Aprendizagem.

meta-analytic integration. Basic and Applied Social Psychology, 12, 3-23.

OSBORN, Alex. How to "Think Up". London:

McGraw-Hill Book Co., 1942.

OSBORN, Alex. Applied imagination:

Principles and procedures of creative thinking.

New York: Charles Scribner's Sons, 1953.

OSBORN, Alex. Applied imagination:

Principles and procedures of creative problem solving (2nd Revised Edition). New York: Charles Scribner's Sons, 1957.

OSBORN, Alex. Applied imagination:

Principles and procedures of creative problem solving (3rd Revised Edition). New York, NY:

Charles Scribner's Sons, 1963.

OSBORN, Alex. O Poder Criador da Mente: Princípios e Processos do Pensamento Criador e do "Brainstorming". Tradução de E. Jacy

Monteiro. $5^{\text {a }}$ edição. São Paulo: IBRASA, 1975.

PARNES, S. J. \& Meadow, A. Effects of "Brainstorming" instructions on creative problem solving by trained and untrained subjects. Journal of Education Psychology, 30: 171-176. 1959.

Paulus et al (2006, 2011).Effects of Task

Instructions and Brief Breaks on

Brainstorming. Group Dynamics: Theory,

Research and Practice 2006, Vol.10. N³ 206-219.

Rossiter e Lilien (1994). New "Brainstorming Principles". Australian Journal of Management, 19, 1, June 1994, The University of New South Wales. 